\title{
Non Linearities \& Dispersion Management in Advanced Communication
}

\author{
N. S. Murti Sarma ${ }^{1}$, S. P. Venu madhava Rao ${ }^{2}$ \\ Sreenidhi Institute of Science and Technology, Yamnampet, Hyderabad-501 301, India
}

\begin{abstract}
Optical communication is identified as one of the advanced communications systems. Dispersion \& non-linear effects cause severe waveform distortion in single channel as well as Wavelength Division Multiplexing (WDM) systems. Of Two methods like Disperion Compensation Fiber and Optical phase compensation, Results are reported for dispersion Compensation fiber.
\end{abstract}

Keywords: Dispersion, WDM, compensation, non linear effects, signle channel

\section{Introduction}

Among the various communication media such as coaxial cables, twisted pairs, optical fibers, radio frequency, satellites, optical fiber is the only medium with enormous bandwidth to handle the rapidly growing high capacity backbone into the world today. The optical fiber is often seen as a perfect transmission medium with almost limitless bandwidth. However in practice, the propagation through optical fiber is the best with several limitations especially as distance is increased to multi span amplified systems [1]. As the transmission systems evolved to longer distances and higher bit rates, the linear effects of fibers i.e. attenuation and dispersion become the important limiting factors. As per WDM systems that transmit multiple wavelengths simultaneously at even higher bit rates and distances, the non linear effects in the fiber begin to present a serious limitation.

The success of high bit rate long haul point to point optical transmission networks depends upon how best the linear and non linear effects are managed. The major linear effects include group velocity dispersion (GVD) of standard single mode fiber, fiber loss, adjacent channel X-talk, polarization mode dispersion (PMD), accumulated ASE noise etc. The non linear affects on the other hand include self phase modulation (SPM), cross phase modulation (XPM), stimulated Brillouin scattering (SBS), stimulated Raman scattering (SRS) and four wave mixing (FWM). [2]

\section{Description of the Methods Used in For Non Linear \& Dispersion Compensation}

The first step towards dispersion management was to develop some suitable fiber having low dispersion. Then the next issue is the dispersion slope management in high bit rate single channel systems. The dispersion arrangement, which considers both the second and third order dispersion characteristics, is shown in the Fig.1. For second order dispersion control, we arrange the PDF (dispersion value D2p, length is $\mathbf{L p}$ ) and NDF (D2n and Ln) so that the subtotal of their product in each repeater span i.e., is not zero but remains a constant value. This is because the local second order dispersion value should be as large as possible to reduce the inter channel interaction caused by non linearity. The system composed of M spans of sets of PDF and NDF and the length of PDF for dispersion compensation is given by $\mathrm{Lp}$ then the second order dispersion value of a unit dispersion management span is expressed as [3]

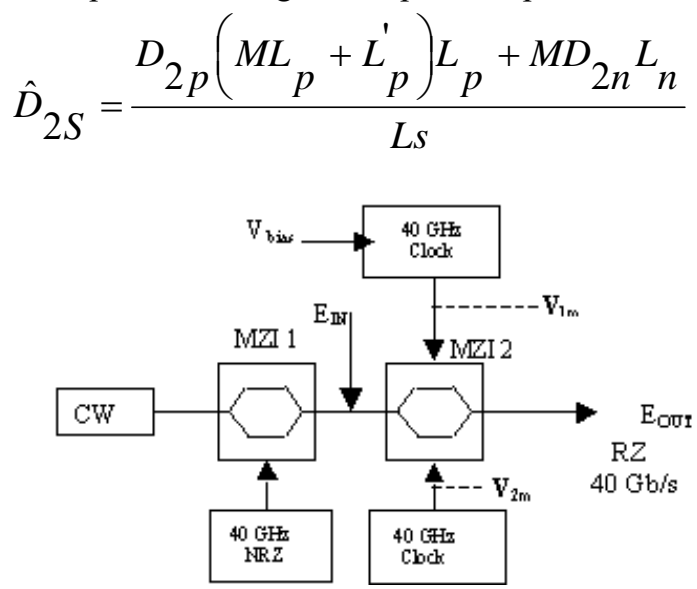

Figure 1: Generation of $40 \mathrm{~Gb} / \mathrm{s} \mathrm{CSRZ}$

The second order dispersion value before compensation by $\mathrm{PDF}$ is given by

$$
\hat{D}_{2 S}=\frac{D_{2 p} L_{p}+D_{2 n} L_{p}}{L_{p}+L_{n}}
$$

which would typically be minus a few $\mathrm{ps} / \mathrm{nm} / \mathrm{km}$ for effective suppression of inter channel interference. Fig2, shows the schematic of SMF and DCF. Its dispersion map is expemplified as below.
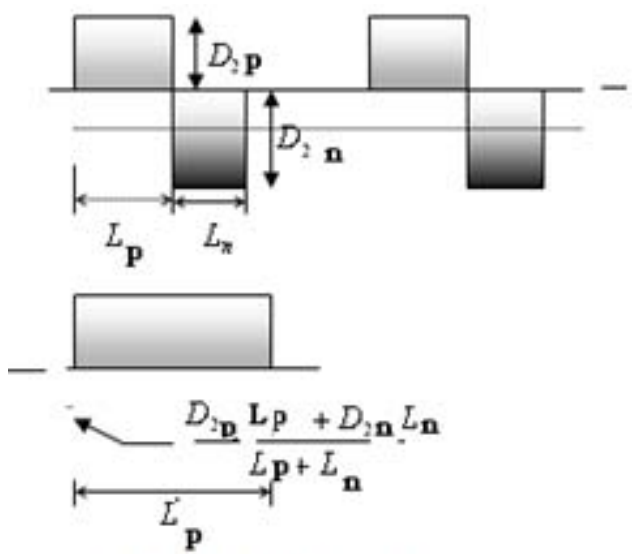

Figure 3 Dispersion map for the $D C F$ compensation scheme 


\section{International Journal of Science and Research (IJSR) \\ ISSN (Online): 2319-7064}

Index Copernicus Value (2013): 6.14 | Impact Factor (2014): 5.611

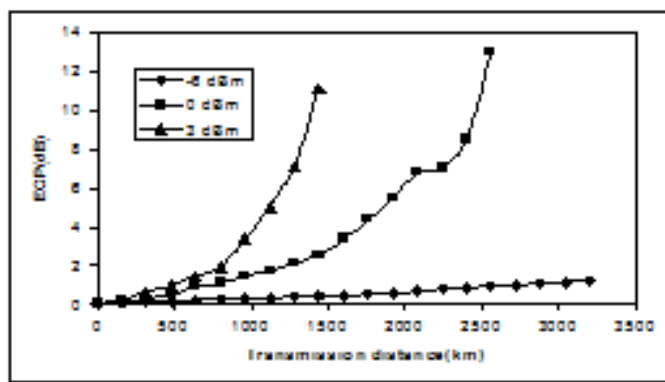

Figure 4: Eye opening penalty vs Transmission distance for NRZ signal for different average input power
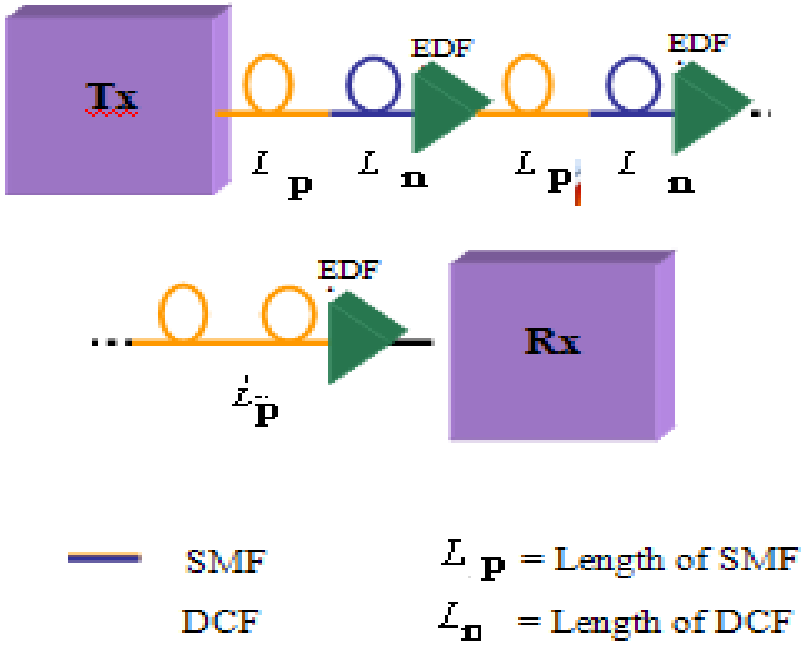

Figure: 2 Sehematic of SM and DCF

The third order dispersion value of the span Ls is given by

$$
D 3 s=\frac{D_{3 P}\left(M L_{p}+L_{p}^{\prime}\right)+M D_{3 n} L_{n}}{L_{s}}
$$

To suppress significant waveform distortion, the lengths of PDF and NDG fibers should be chosen to satisfy the following relations

$$
\begin{aligned}
& L_{D 2 p}=\frac{T_{0}^{2}}{\beta_{2 p}}, L_{N L p}=\frac{1}{\gamma_{p P}}, \\
& L_{D 2_{n}}=\frac{T_{0}^{2}}{\beta_{2 n}}, \\
& \text { and } L_{N L n}=\frac{1}{\gamma_{n} P_{n}},
\end{aligned}
$$

Are the dispersion and nonlinear lengths of PDF and NDF respectively.

\section{Results and Discussion}

\section{Volume 5 Issue 2, February 2016} single channel DCF compensation for different input powers at a transmission distance of $320 \mathrm{~km}$ 


\section{International Journal of Science and Research (IJSR) \\ ISSN (Online): 2319-7064}

Index Copernicus Value (2013): 6.14 | Impact Factor (2014): 5.611

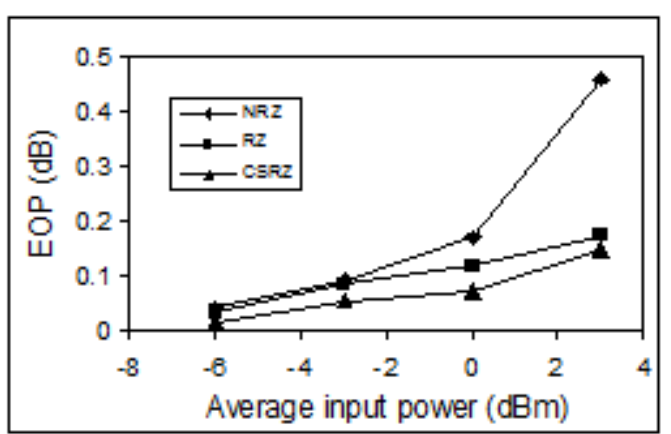

(a)

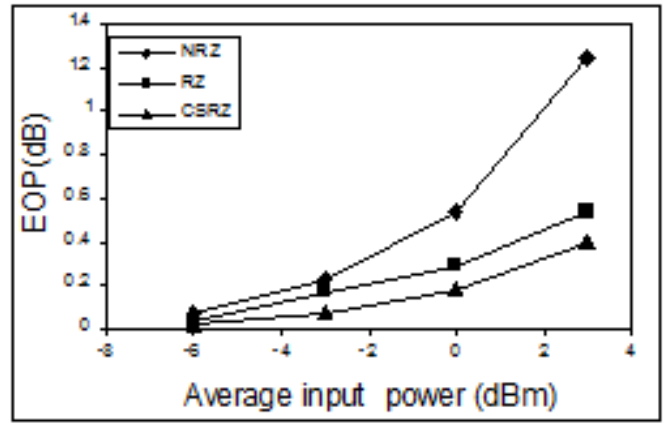

(b)

Figure 9: Comparison of different modulation formats with respect to EOP in a $4 \times 40 \mathrm{Gbps}$ transmission system employing DCF compensation with channel spacing of $100 \mathrm{GHz}$ for a transmission distance of $320 \mathrm{~km}(\mathrm{a})$, and 800 $\mathrm{km}(\mathrm{b})$

The results generated using MATLAB are reported from Fig.4 to Fig.9. Eye opening penality of NRZ is comparatively moderate for $0 \mathrm{~dB}$ average output power. For RZ signal as shown in Fig.5, the slope found to be progressively decreased. Fig6 shows the decrement of slope of the EOP with signal power. However, $9 \mathrm{dBm}$ power shows the sustainability for longer distances. Fig.7 highlights the CSRZ technique that has very small slope compared with others. The same is confirmed in Fig8 for a limited distance of $320 \mathrm{Km}$ transmission distance.Fig.9, compares the same for $800 \mathrm{Km}$ also. This shows the nearness of CSRZ and RZ format.

\section{Conclusions}

The compensation of dispersion and dispersion slope using DCF is studied. This type of compensation technique works well for lower input powers. As this compensation technique does not compensate non linearity, it shows layer eye opening penalty at higher input powers. Also as the nonlinear coefficients of the dispersion coefficient fiber is high, the nonlinearity is more. For this the power launched into the DCF is properly chosen. Here in our simulations we have compensated the total loss over the PDF by using EDFA and it is launched into the DCF. This compensation is economically suited for short range application.

\section{References}

[1] G.P Agarwal, "Non linear Fiber optics", Academic press, 1989
[2] B.W.Hakki, "polarization mode dispersion in a single mode fiber", J.light Wave technol.,1996,vol.14.,PP.2202-2208

[3] D.Brever and K.petermann, "Comparison of NRZ and RZ- modulation format for 40Gbps FDM standard fiber systems", IEEE photon Technol.lette.,vol.9,pp.98-400, March 1997

[4] W.H.Loh,F.Q.Zhou,J.J.Pan,ECEC'99,pp.162-163

[5] S.Watanabe, M.Shirsasaki, "Exact compensation for both chromatic dispersion and Kerr effect in a fiber using optical phase conjugation", IEEE J.Light wave Technol, vol 14, pp.243-248, March 1996 